\title{
Interdigital Tinea: The Forerunner of Infectious Eczematoid Dermatitis
}

\author{
Kyle R. Bhatia ${ }^{1}$, Robert T. Brodell, MD², Ashish C. Bhatia, MD², Chelsea S. Mockbee, MD² \\ ${ }^{1}$ Loyola University Chicago, Stritch School of Medicine Maywood, IL \\ 2Department of Dermatology, University of Mississippi Medical Center Jackson, MS \\ ${ }^{3}$ Department of Dermatology, Northwestern University, Chicago, IL
}

\section{ABSTRACT}

Infectious eczematoid dermatitis (IED) is defined as an acute, eczematous eruption that occurs secondary to autosensitization to purulent drainage from a primary infected site. The condition is believed to develop when bacterial products, most often the result of Staphylococcal or Streptococcal species, act as haptens and stimulate an immune response. IED typically manifests as a plaque with associated vesicles and pustules surrounding drainage from a central infectious source, or as oozing, erythema, crusting, and scaling spreading peripherally from a central infectious source. Management of IED includes both targeting the causative primary infection and suppressing the immune response producing a hypersensitivity reaction. This report details two cases. Case 1 describes a common presentation of tinea pedis. Case 2 is that of a 28 year-old-male who presented with an acute onset tender, pruritic, weeping rash after wearing boots for two straight days, and who was subsequently diagnosed and treated for IED.

\section{CASE PRESENTATION}

Case 1 is a male patient who presented with chronic scaling of the pedal interdigital spaces associated with mild intermittent pruritus. (Figure $1 \mathrm{~A}$ and $1 \mathrm{~B})$.

Case 2 is of a 28 year-old-male that presented with an acute onset erythematous eruption bilaterally on the feet. He had a long history of mildly pruritic and intermittently painful cracking between the fourth and fifth toes with associated thick, moist scaling. During a hunting trip, he walked through wetlands and wore his boots for two straight days, even while sleeping. When the boots were removed, he discovered a bilateral, tender, pruritic, weeping rash across his toes and distal foot. On physical exam, a well-demarcated, erythematous, oozing, edematous eruption with erosion was present on the plantar and dorsal surface of the feet and toes (Figure 2A and 2B).

Figure 1. (A) Scaling and erythema of the interdigital skin typical of tinea pedis.

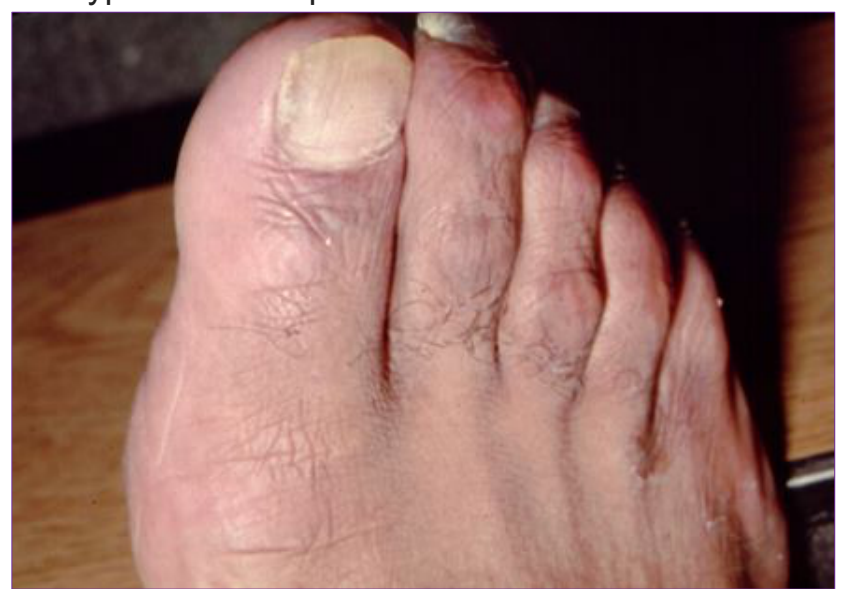

July 2020 Volume 4 Issue 4 


\section{SKIN}

Figure 1. (B) Scaling of subdigital skin typical of tinea pedis.

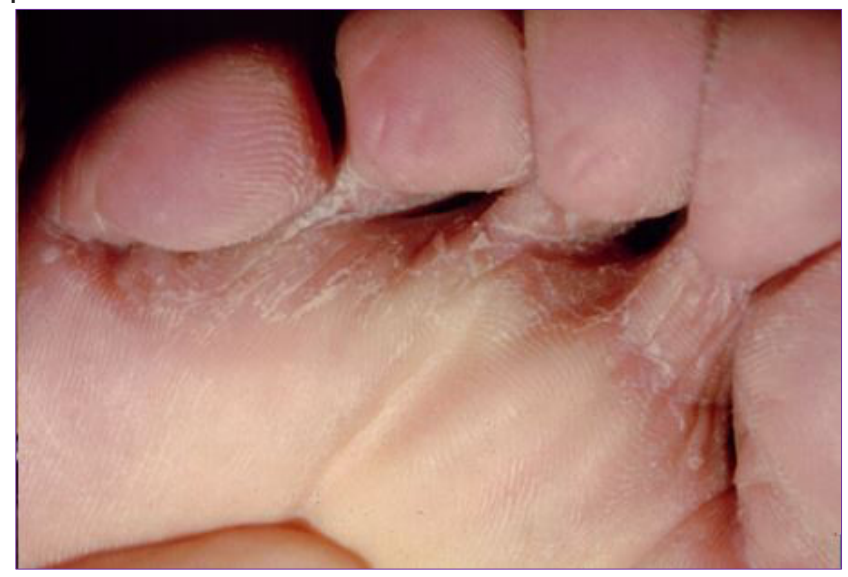

Figure 2. Oozing, erythematous eruption with interdigital and subdigital purulence overlying edematous toes of a male patient after two straight days without removing boots.
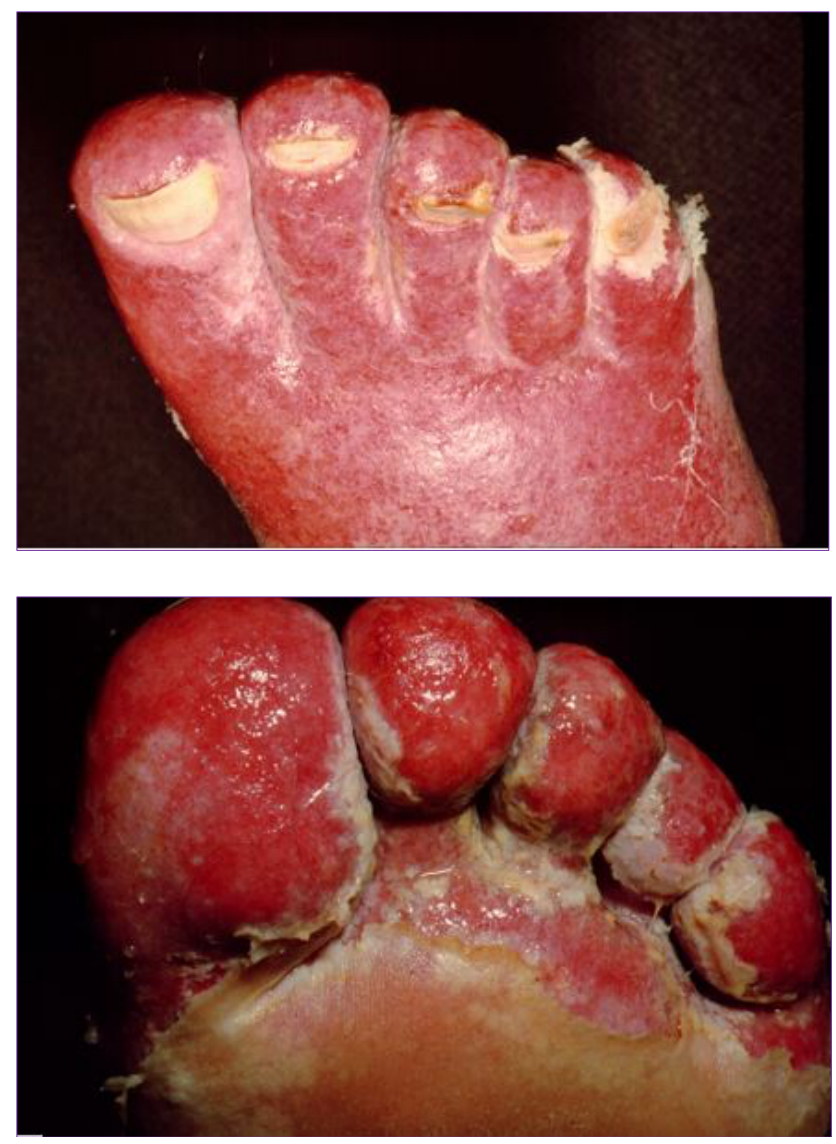

Table 1. Diagnostic Criteria for Infectious Eczematoid Dermatitis as Proposed by Yamany and Schwartz ${ }^{7}$. (C) 2014 European Academy of Dermatology and Venereology - Reproduced with permission from John Wiley and Sons.

\begin{tabular}{|c|c|}
\hline & Clinical Characteristics \\
\hline $\begin{array}{c}\text { Major Criteria } \\
\text { (2 of } 2 \text { must be } \\
\text { present) }\end{array}$ & $\begin{array}{l}\text { 1. A primary infected lesion } \\
\text { with purulent drainage. } \\
\text { 2. Eruption spreading } \\
\text { peripherally from primary } \\
\text { lesion. }\end{array}$ \\
\hline $\begin{array}{c}\text { Minor Criteria } \\
\text { (1 of } 2 \text { must be } \\
\text { present) }\end{array}$ & $\begin{array}{l}\text { 1. Peripheral vesicles and } \\
\text { pustules with central } \\
\text { oozing, crusting, and } \\
\text { scaling. } \\
\text { 2. Oozing, crusting, and } \\
\text { scaling throughout entirety } \\
\text { of eruption. }\end{array}$ \\
\hline
\end{tabular}

\section{DISCUSSION}

In case 1 , the patient had a symbiotic relationship with a non-complicated tinea pedis infection, with fungal culture revealing Trichophyton rubrum. Tinea pedis is the most common dermatophytosis, affecting up to $25 \%$ of the population in the United States. It is most commonly associated with Trichophyton rubrum, though Epidermophyton, Microsporum, and other Trichophyton species have also been implicated. ${ }^{1}$ Though dermatophytes are viewed as the causative organisms of tinea pedis, associated overgrowth of coinhabiting bacterial species is common, especially in more severe disease states. ${ }^{2}$ As disease severity increases, fungi are less frequently recoverable: fungi were demonstrated in $84 \%$ of mild and asymptomatic tinea pedis cases; $55 \%$ of moderately symptomatic cases; and $36 \%$ of severe cases. In these severe cases, increased rates of overgrowth of various bacterial species, including grampositive Staphylococcus species and gramnegative Proteus were found. ${ }^{3}$ These interdigital bacteria have higher rates of July 2020 Volume 4 Issue 4 
antibiotic resistance, which can be attributed to dermatophyte production of penicillin-like and streptomycin-like antibiotic substances. ${ }^{4,5}$ Clinical presentation of intertriginous tinea pedis infection is highly variable. Erythema, fissures, and pruritus may be present between the $4^{\text {th }}$ and $5^{\text {th }}$ toes, though some patients may be entirely asymptomatic. Tinea pedis is typically treated with topical antifungals and hygiene measures to keep affected areas clean and dry. Systemic antifungals may be used in severe or treatment-resistant cases. ${ }^{6}$ As the tinea is cleared, the bacterial component usually resolves without specific treatment.

In case 2, a bacterial culture revealed Staphylococcus aureus, while fungal culture was negative likely due to bacterial suppression of fungal growth. This patient's presentation is consistent with infectious eczematoid dermatitis (IED). This condition is defined as an acute, eczematous eruption that occurs secondary to autosensitization to purulent drainage from a primary infected site. ${ }^{7}$ In a 1902 study of 1200 new patients to an outpatient dermatology clinic, Engman reported that 35 , or $2.9 \%$, had presentations consistent with IED. ${ }^{8}$ A study at the Mayo Clinic examining inpatient dermatology admissions from 2000-2010 reported similar results, finding that $2.9 \%$ of 2216 admissions were due to IED. ${ }^{9}$ IED is believed to develop when purulent drainage from a primary infection causes autosensitization, with hypersensitivity developing as bacterial products act as haptens and stimulate an immune response. ${ }^{7}$ Staphylococcal and/or Streptococcal species are most commonly associated with IED. ${ }^{10}$ In this case, interdigital tinea pedis, and associated bacterial infection, flourished in the warm, moist environment of the patient's wet boots over two days. An immune response stimulated the subsequent acute, eczematous eruption.

The differential diagnosis for case 2 includes cellulitis and contact dermatitis. The clinical presentation of a pruritic and oozing, erythematous eruption was not consistent with cellulitis. Though the eczematous eruption may have been attributed to contact dermatitis, the patient did not have a history of allergic contact dermatitis to leather or any other contactants. IED accounts for all aspects of the patient's presentation, namely the initial fungal infection, secondary bacterial infection, and autosensitization and subsequent eczematous response.

It is important to note that IED is distinct from auto-eczematization (dermatophytid or id reaction), a generalized process in which vesicles or pustules appear at distant sites in a patient with a localized tinea infection. These dermatophytid reactions may be the only presenting sign in an otherwise asymptomatic patient and typically resolve with treatment of the primary tinea infection. ${ }^{11}$ IED is a localized process that occurs adjacent to and surrounding the primary infectious locus. ${ }^{7}$

IED often initially presents as a plaque with associated vesicles and pustules surrounding a central source of infectious drainage. The lesion may spread peripherally, and older areas are distinguished by oozing, erythema, scaling, and crusting. ${ }^{7}$ Alternatively, patients may present with oozing, erythema, crusting, and scaling that spreads peripherally from the central infectious source but with no evidence of vesicles or pustules. ${ }^{8}$ The patient from case 2 presented as the latter type. Though IED can affect any part of the body, it is commonly found on the ears in patients with otitis externa, on the face and the nares in children with nasal July 2020 Volume 4 Issue 4 
Staphylococcus, and on the lower extremities in adults. ${ }^{12}$ In a 2015 comprehensive review of IED, Yamany and Schwartz proposed the major and minor diagnostic criteria found in Table 1. The patient from case 2 met both the major criteria and the second minor criteria.

The management of IED requires targeting the causative infection and suppressing the immune response producing a type IV hypersensitivity reaction. In addition, local wound care should be optimized. The patient's primary tinea pedis infection was treated with oral terbinafine $250 \mathrm{mg}$ daily for 2 weeks. Because of the severity of this condition, the secondary Staphylococcal aureus infection was treated with oral doxycycline $100 \mathrm{mg}$ twice daily for 10 days. In order to quell the associated allergic contact dermatitis, the patient was placed on oral prednisone $60 \mathrm{mg}[1 \mathrm{mg} / \mathrm{kg}$ ], which was tapered off over 2 weeks. Substantial improvement was noted in just 24 hours. Local skin care included Burrow's solution applied three times daily, and the application of mupirocin ointment to promote barrier protection and fight superficial bacterial infection. ${ }^{7}$ The patient's feet showed only post inflammatory erythema at 2 weeks. Ketoconazole cream weekly between the toes was recommended to prevent recurrent tinea.

\section{Conflict of Interest Disclosures: None}

Funding: None

\section{Corresponding Author:}

Robert T. Brodell, MD

106 Chadwyck Place

Madison, MS 39110

Phone: $330-883-5302$

Fax: 601-984-1150

Email: rbrodell@umc.edu

\section{References:}

1. Ilkit M, Durdu M. Tinea pedis: the etiology and global epidemiology of a common fungal infection. Crit Rev Microbiol. 2015;41(3):374388. doi:10.3109/1040841X.2013.856853

2. Gupta AK, Skinner AR, Cooper EA. Interdigital tinea pedis (dermatophytosis simplex and complex) and treatment with ciclopirox $0.77 \%$ gel. Int J Dermatol. 2003;42 Suppl 1:23-27. doi:10.1046/j.1365-4362.42.s1.1.x

3. Leyden JJ, Kligman AM. Interdigital athlete's foot. The interaction of dermatophytes and resident bacteria. Arch Dermatol. 1978;114(10):14661472. doi:10.1001/archderm.114.10.1466

4. Youssef N, Wyborn CH, Holt G. Antibiotic production by dermatophyte fungi. J Gen Microbiol. 1978;105(1):105-111. doi:10.1099/00221287-105-1-105

5. Kates SG, Nordstrom KM, McGinley KJ, Leyden JJ. Microbial ecology of interdigital infections of toe web spaces. J Am Acad Dermatol. 1990;22(4):578-582. doi:10.1016/01909622(90)70075-s

6. Canavan TN, Elewski BE. Identifying Signs of Tinea Pedis: A Key to Understanding Clinical Variables. J Drugs Dermatol. 2015;14(10 Suppl):s42-s47.

7. Yamany T, Schwartz RA. Infectious eczematoid dermatitis: a comprehensive review. J Eur Acad Dermatol Venereol. 2015;29(2):203-208. doi:10.1111/jdv.12715

8. Engman MF. An infectious form of an eczematoid dermatitis. St. Louis Courier of Medicine 1902; 27: 401-414.

9. Storan E, McEvoy M, Wetter D, et al. Experience with the dermatology inpatient hospital service for adults: Mayo Clinic, 2000-2010. J Eur Acad Dermatol Venereol. 2013;27:1360-1365.

10. Slatkin MH. Local use of bacitracin. $J$ Invest Dermatol 1948; 10: 179-188.

11. Weinstein A, Berman B. Topical treatment of common superficial tinea infections. Am Fam Physician. 2002;65(10):2095-2102.

12. McDaniel WE, Tamura J. Auto-sensitization in infectious eczematoid dermatitis. AMA Arch Derm Syphilol. 1950;62(5):703-704. 